\title{
Nanotechnology and Nanomedicine
}

\section{Opinion}

The field of nanotechnology has grown rapidly over the past few years and has even ventured into the field of clinical medicine. Precious metals in nano-size form like $\mathrm{Ag}$ and $\mathrm{Au}$ nanoparticles (NPs) are out of all kinds of nanoparticles that attracted many scientists due to their potential application indeed. The size and morphology of these NPs play a crucial role in predicting their physical properties such as optical and plasmon resonance properties which suggest that they will have multiple applications across both therapeutics and diagnostics in the field of nanomedicine $[1,2]$. Modern nanomedicine nowadays has begun evaluating the use of (Au-NPs) in medicine and many specific functional studies are addressing issues such as potential toxicity [3], cellular trafficking [4-7], optimization in building multicomponent nano-constructs, their efficacy for gene regulation [8] and drug delivery [6], their pharmacokinetics [9] and even their application as adducts in photo-thermal therapies $[10,11]$. It appears that the interaction of protein containing biological fluids with these (Au-NPs) is readily adsorbs or may form covalent bonding to the surface of these (Au-NPs) which is large compared to their volume suggest their use with the antibodies for use in the treatment of cancer cells.

Silver (Ag) for a long time has been as effective antibacterial agent against both aerobic and anaerobic bacteria. The use of (Ag-NPs) seems to have reduced cellular toxicity but not antibacterial efficacy [12]. Indeed, Kim et al. [12] demonstrated clearly that the superior antibacterial properties of (Ag NPs), are due to the formation of free radicals from the surface of Ag. There are seven characteristics for silver sterilization (Broad spectrum antimicrobial, Potent sterilization, Permeability is strong, Repair regeneration, Antibacterial lasting, Safe and nontoxic, and no drug resistance). Furthermore, the addition of antibiotics to (Ag-NPs) has been shown to have synergistic effects against micro-organisms [13]. Strydom et al. [14] demonstrated that modification of silver sulphadiazine using dendrimers increased the anti-bacterial efficacy. (Ag-NPs) shows a direct anti-inflammatory affects and improved the healing process significantly when compared with controls apart from being an excellent antibacterial agent.

\section{Conclusion}

We have seen that the advances in nanotechnology enabled us to deal with precious metal nanoparticles, which would appear to satisfy many beneficial aspects for clinical use. Nonetheless, it is still far too early to say that the panacea is already here. Nanomedicine, is expected to be involve all aspects of medicine including synthesis, bioavailability, biodistribution of nanomedicines, delivery, pharmacodynamics, and pharmacokinetics of nanomedicines; imaging; diagnostics; improved therapeutics; innovative biomaterials; interactions of nanomaterials with cells, tissues, and living organisms; regenerative medicine; public health; toxicology; point of

Opinion
Volume 7 Issue 1 - 2018
Zuheir El-bayyari*
Department of Basic Sciences and Mathematics, Philadelphia
University, Jordan
*Corresponding author: Zuheir El-bayyari, Department
of Basic Sciences and Mathematics, Faculty of Science,
Philadelphia University, Aein Albasha 19392, Jordan,
Email: zbayyari@rocketmail.com
Received: January 18, 2018 | Published: February 16, 2018

care monitoring; nutrition; nanomedical devices; prosthetics; biomimetics; and bioinformatics.

\section{Acknowledgment}

None.

\section{Conflicts of Interest}

None.

\section{References}

1. El-bayyari Z (1998) Molecular Dynamics Computer Simulations of Silver Aggregates (Agn; $\mathrm{n}=3-13$ ) : Empirical Many - Body Potential Energy Function Calculation Phys Stat Sol 208: 339-347.

2. El-Bayyari Z (2001) Gold Microclusters (Aun; $n=3-13$ ): A Molecular - Dynamics Computer Simulation. In "Computer Simulation Studies in Condensed Matter Physics XIV. In: Landau DP \& Lewis SP (Eds.), Germany, pp. 47.

3. Papasani MR, Wang GK, Hill RA (2012) Gold nanoparticles: the importance of physiological principles to devise strategies for targeted drug delivery. Nanomedicine 8(6): 804-814.

4. Sadauskas E, Danscher G, Stoltenberg M, Vogel U, Larsen A, et al. (2009) Protracted elimination of gold nanoparticles from mouse liver. Nanomedicine 5(2): 162-169.

5. Lin IC, Liang M, Liu TY, Monteiro MJ, Toth I (2012) Cellular transports pathways of polymer coated gold nanoparticles. Nanomedicine: Nanotechnology, Biology and Medicine 8(1): 8-11.

6. Gu Y-J, Cheng J, Man CW-Y, Wong W-T, Cheng SH (2012) Golddoxorubicin nanoconjugates for overcoming multidrug resistance. Nanomedicine: Nanotechnology, Biology, and Medicine 8(2): 204211.

7. Wang G, Norton AS, Pokharel D, Song Y, Hill RA (2013) KDEL peptide gold nanoconstructs: promising nanoplatforms for drug delivery. Nanomedicine: Nanotechnology, Biology and Medicine 9(3): 366374.

8. Sharma A, Tandon A, Tovey JC, Gupta R, Robertson JD, et al. (2011) Polyethylenimine-conjugated gold nanoparticles: Gene transfer potential and low toxicity in the cornea. Nanomedicine: Nanotechnology, Biology and Medicine 7(4): 505-513. 
9. Simpson CA, Salleng KJ, Cliffel DE, Feldheim DL (2013) In vivo toxicity, biodistribution, and clearance of glutathione-coated gold nanoparticles. Nanomedicine 9(2): 257-263.

10. Letfullin RR, Iversen CB, George TF (2011) Modeling nanophotothermal therapy: kinetics of thermal ablation of healthy and cancerous cell organelles and gold nanoparticles. Nanomedicine 7(2): 137-145.

11. Raoof M, Corr SJ, Kaluarachchi WD, Massey KL, Briggs K, et al. (2012) Stability of antibody-conjugated gold nanoparticles in the endolysosomal nanoenvironment: implications for noninvasive radiofrequency-based cancer therapy. Nanomedicine 8(7): 10961105.
12. Kim JS, Kuk E, Yu KN, Kim JH, Park SJ, et al. (2007) Antimicrobial effects of silver nanoparticles. Nanomedicine 3(1): 95-101.

13. Dar MA, Ingle A, Rai M (2013) Enhanced antimicrobial activity of silver nanoparticles synthesized by Cryphonectria sp. evaluated singly and in combination with antibiotics. Nanomedicine 9(1): 105110.

14. Strydom SJ, Rose WE, Otto DP, Liebenberg W, de Villiers MM (2013) Poly (amidoamine) dendrimer-mediated synthesis and stabilization of silver sulfonamide nanoparticles with increased antibacterial activity. Nanomedicine 9(1): 85-93. 